\title{
A systematic review of non-pharmacological interventions for BPSD in nursing home residents with dementia: from a perspective of ergonomics
}

\author{
Gubing Wang, ${ }^{1}$ (1) Armagan Albayrak, ${ }^{1}$ and Tischa J. M. van der Cammen ${ }^{1,2,3}$ \\ ${ }^{1}$ Faculty of Industrial Design Engineering, Delft University of Technology, Delft, the Netherlands \\ ${ }^{2}$ Section of Geriatric Medicine, Department of Internal Medicine, Erasmus MC, University Medical Center Rotterdam, Rotterdam, the Netherlands \\ ${ }^{3}$ Academic Department of Geriatric Medicine, Brighton and Sussex Medical School, Brighton, UK
}

ABSTRACT

Background: Non-pharmacological interventions for Behavioral and Psychological Symptoms of Dementia (BPSD) have been developed; however, a systematic review on the effectiveness of this type of intervention from a perspective of ergonomics is lacking. According to ergonomics, the capabilities of Persons with Dementia (PwD) should be considered in the interventions for the outcomes to be reliable. We aimed to systematically review the non-pharmacological interventions for BPSD in nursing home residents with an additional assessment criterion based on ergonomics, specifically, capability consideration.

Methods: The electronic databases MEDLINE, EMBASE, and PsycINFO were searched for nonpharmacological interventions treating BPSD in nursing homes. The interventions were categorized according to the capabilities of $\mathrm{PwD}$ required to participate. Study quality was assessed by National Health and Medical Research Council (NHMRC) evidence hierarchy and the capability consideration.

Results: Sixty-four clinical trials met the inclusion criteria; 41 trials reported a significant reduction in at least one BPSD symptom; 20 trials reported no significant reduction in BPSD symptoms; three trials reported adverse effects after the intervention. Interventions were categorized into sensory-, cognition-, and movementoriented. Capabilities of PwD were not considered in 28 trials, especially for sensory capabilities.

Conclusions: The majority of the clinical trials reported a significant reduction in BPSD. The quality of evidence for nonpharmacological interventions in these trials is low due to the lack of capability consideration, data inhomogeneity, and inadequate study design and reporting. Future studies should focus on improving the quality of evidence by including capability consideration and examining if a relationship between capability consideration and effectiveness of non-pharmacological interventions exists.

Key words: behavioral and psychological symptoms of dementia, behavioral therapy, nursing homes, aging, dementia

\section{Introduction}

Over $80 \%$ of Persons with Dementia (PwD) will develop Behavioral and Psychological Symptoms of Dementia (BPSD), also referred to as neuropsychiatric symptoms, during the course of their disease (Abraha et al., 2017), with 97\% of PwD developing at least one symptom over a five-year period (Steinberg et al., 2008). BPSD is defined as "signs and symptoms of disturbed behavior, mood, thought, or perception" (Kales et al., 2015). The Neuropsychiatric

Correspondence should be addressed to: Gubing Wang, Faculty of Industrial Design Engineering, Delft University of Technology, Building: 32, Room C-3-080, Landbergstraat 15, 2628 CE Delft, the Netherlands. Phone: +31(0)15 27 85039; Fax: +31 (0)15 27 87179. Email: g.wang-2@tudelft.nl. Received 12 Jun 2018; revision requested 30 Jul 2018; revised version received 10 Aug 2018; accepted 03 Sep 2018. First published online 18 October 2018.
Inventory-Questionnaire (NPI-Q) has categorized the reported signs and symptoms into: delusions, hallucinations, agitation/aggression, depression/ dysphoria, anxiety, elation/euphoria, apathy/indifference, disinhibition, irritability/lability, motor disturbance, nighttime behaviors, and disturbance in appetite/eating (Kaufer et al., 2000). BPSD could induce physical injuries and psychological distress in PwD and their caregivers, and it is identified as a predictor of nursing home placement (Gaugler et al., 2003). In nursing homes, caregivers have reported to respond to PwD exhibiting BPSD with verbal or physical abuse or to minimize contact with them, thus reducing the quality of care received by $\mathrm{PwD}$ (Kales et al., 2015). Nursing home administrators could face increased financial 
costs due to increased medical and psychological care, security concerns, and staff turnover (Kales et al., 2015). Therefore, effective management of BPSD needs to be identified and applied, especially in the nursing home setting.

Antipsychotic medication has been applied to manage BPSD, which has been shown to have low efficacy and serious side effects, such as increasing fall risk, mortality rate, and stroke occurrence (Gill et al., 2007; Seppala et al., 2018). As a result, non-pharmacological interventions have been developed to act as an alternative of antipsychotic medication. This study proposes a hypothesis that new insights could be gained if the non-pharmacological interventions and their clinical trials are also reviewed and assessed from a perspective of ergonomics. Ergonomics studies "the interactions between humans and other elements of a system, and applies theories, principles, data, and methods to design for optimizing human well-being and system performance" (Karwowski, 2012). Human performance depends on the person's capabilities and limitations; while the system has its own requirements and affordances. These requirements and affordances need to match with the capabilities and limitations of the target population for the outcome of the system to be reliable. From an ergonomist's view, nonpharmacological interventions could be regarded as the system. The remaining capabilities of PwD and the requirements of the interventions should match for the outcome of the clinical trial to be reliable. Capability is an umbrella term used in ergonomics to describe one's ability in sensory, cognition, and movement aspects when interacting with a system (Czaja and Nair, 2006). One needs certain levels of capabilities to be able to perform certain cognitive and functional activities, namely Activities of Daily Living (ADL) or Instrumental Activities of Daily Living (IADL).

Several systematic reviews have reviewed and assessed the quality of the clinical trials to conclude on the quality of evidence on the effectiveness for each intervention. Abraha et al. found that music therapy and behavioral management techniques are effective in managing BPSD, in general, while the evidence base is weak due to variations in application of the interventions and measurements (Abraha et al., 2017). Brasure et al. reached the same conclusion on the weak evidence base after systematically reviewing non-pharmacological interventions in managing agitation and aggression in $\mathrm{PwD}$ (Brasure et al., 2016). Cohen-Mansfield identified that many non-pharmacological interventions have led to a statistically and clinically meaningful improvement in the management of behavioral problems, and stated that the variation in criteria for success, screening procedures, and control procedures together with underreported treatment failures prevent further conclusions to be drawn (Cohen-Mansfield, 2001). A meta-analysis showed that non-pharmacological interventions delivered by family caregivers had an effect size in managing BPSD at least equaling that of antipsychotic medication (Brodaty and Arasaratnam, 2012). However, as the focus of the current review is on capability considerations of $\mathrm{PwD}$ when they are interacting with non-pharmacological interventions, caregiver interventions are not included in this review as caregiver interventions do not require capabilities of PwD.

The systematic reviews currently available in the literature have evaluated neither the nonpharmacological interventions nor their clinical trials based on the abovementioned capability matching principle in ergonomics. Specifically, these reviews have not assessed if capabilities of PwD were considered in the non-pharmacological interventions and their clinical trials (Abraha et al., 2017; Brasure et al., 2016; Cohen-Mansfield, 2001). In addition, the availability of resources, severity of cognitive impairment, and levels of comorbidity are different in community and nursing home settings. However, no systematic review on non-pharmacological interventions for BPSD has distinguished between interventions in community settings and nursing home settings. A systematic review on the effectiveness of non-pharmacological interventions for BPSD in nursing home residents is also lacking. Therefore, this study aims to systematically review the nonpharmacological interventions for BPSD in nursing home residents, with a special focus on capability considerations as recommended in ergonomics.

\section{Methods}

\section{Search strategy}

The literature search was performed in three electronic databases: PsycINFO, EMBASE, and MEDLINE. For the searches, the following sets of search terms were used: (1) dementia, (2) BPSD, and (3) nursing home, with the searches limited to therapy (maximize sensitivity). The first set of terms included "dementia (exploded)", "dementi*", "Alzheimer*". The second set of terms consisted of "neuropsych*", "behav*", "behav* problems (exploded)". The third set of terms involved "nursing home (exploded)", "nursing care". The date of the last search is January 8, 2018. The full search strategy is listed in the supplemental digital content (SDC) -1 (published as supplementary material online attached to the electronic version of this paper at https://www.cambridge.org/core/journals/ international-psychogeriatrics). 


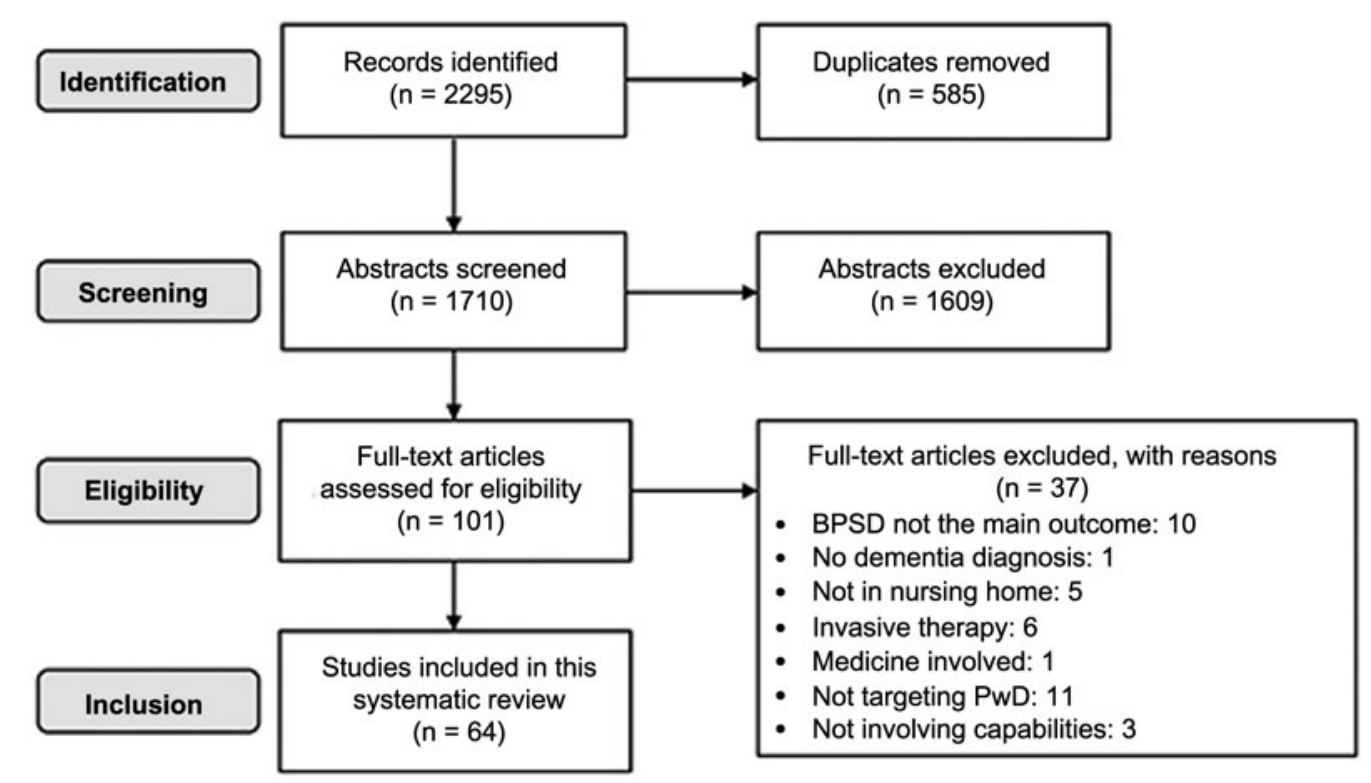

Figure 1. Flow diagram of the systematic review.

\section{Inclusion criteria}

Inclusion criteria for studies relevant for this review were: (1) studies that involved nursing home residents with a diagnosis of dementia; (2) studies that applied non-pharmacological interventions for BPSD which require capabilities of nursing home residents; (3) studies that reported on effects of the interventions on symptoms of BPSD; (4) studies with a pre-post, (quasi) experimental, cross-sectional, randomized controlled, or longitudinal design; and (5) studies written in English and published in a peer-reviewed journal between January 1, 1998 and January 1, 2018.

\section{Intervention categorization}

The interventions were categorized according to the capabilities of PwD required to participate. Ergonomics has investigated human capabilities and how these capabilities change with age. The age-related capabilities investigated were in terms of the sensory, cognition, and movement aspects (Freudenthal, 1999). As the majority of PwD are over the age of 65 , they suffer from capability declines in these three aspects not only due to dementia, but also age. Therefore, the interventions were categorized into sensory-, cognition-, and movement-oriented in this review.

\section{Quality assessment}

We evaluated the quality of each trial based on the National Health and Medical Research Council (NHMRC) evidence hierarchy (NHMRC, 2000). In addition, each trial was judged for whether capabilities of $\mathrm{PwD}$ were considered. Two criteria were used in this review for rating "capability consideration." First, we assessed if the intervention investigated in the trial had been designed with the capabilities of PwD in mind; that is, if design guidelines for PwD had been incorporated or PwD had been involved in the design process. When the first criterion was not satisfied, we assessed if the trial only included PwD with adequate capabilities required by the intervention. For example, if $\mathrm{PwD}$ with hearing impairments were excluded from a clinical trial on Music Therapy. The clinical trial was rated "Yes" for "capability consideration," if it satisfied either criterion, and rated "No" when both criteria were not met.

\section{Results}

The study selection process, guided by Preferred Reporting Items for Systematic Reviews and MetaAnalysis (PRISMA), is shown in Figure 1. The search disclosed 2295 abstracts, of which 101 clinical trials were chosen as potentially relevant; of these, 64 trials met all the inclusion criteria. The summary of the three intervention categories is shown in Table 1. More intervention types were categorized as sensoryoriented, rather than cognition- and movementoriented. The summary of intervention outcomes is shown in Figure 2. Forty-one trials reported a significant reduction on at least one BPSD-symptom; 20 trials reported no significant reduction while three trials reported that the BPSD-symptoms worsened after the intervention. The "capability considerations" of the clinical trials are summarized and shown in Figure 3. As shown in Figure 3, 28 trials did not consider the capabilities of PwD. The details of 
Table 1. Summary of the three intervention categories for BPSD in nursing home residents according to capabilities in ergonomics

\begin{tabular}{|c|c|}
\hline $\begin{array}{l}\text { INTERVENTION } \\
\text { CATEGORIES }\end{array}$ & $\begin{array}{l}\text { INTERVENTION TYPES } \\
\text { (NUMBER OF TRIALS) }\end{array}$ \\
\hline Sensory-oriented & $\begin{array}{l}\text { Music Therapy (21) } \\
\text { Aromatherapy (9) } \\
\text { Light Therapy (5) } \\
\text { Technology-Assisted Therapy (5) } \\
\text { Snoezelen Therapy (2) } \\
\text { Positive Image Therapy (1) } \\
\text { Animal-Assisted Therapy (1) } \\
\text { Clowning Therapy (1) }\end{array}$ \\
\hline Cognition-oriented & $\begin{array}{l}\text { Reminiscence Therapy (5) } \\
\text { Simulated Presence Therapy (3) } \\
\text { Cognitive Stimulation Therapy (1) } \\
\text { Storytelling Therapy (1) }\end{array}$ \\
\hline Movement-oriented & $\begin{array}{l}\text { Exercise Therapy (5) } \\
\text { Outdoor Activity Therapy (4) }\end{array}$ \\
\hline
\end{tabular}

each trial are listed in the supplemental digital content (SDC) -2 (published as supplementary material online attached to the electronic version of this paper at https://www.cambridge.org/core/ journals/international-psychogeriatrics). The details include: trial type, patient type, intervention, scores in NHMRC and "capability consideration," and outcomes. Each intervention type is described below in the sequence of sensory-oriented, cognitionoriented, and movement-oriented categories, with the intervention types that were investigated by only one trial described together as "other interventions" in each category. For trials satisfying the first "capability consideration" criterion (i.e. Snoezelen Therapy, Technology-Assisted Therapy, AnimalAssisted Therapy, Clowning Therapy, and Reminiscence Therapy), we included the specific design guidelines used, or features of the intervention that had been modified based on preliminary field testing in the description below.

\section{Sensory-oriented therapy}

Music Therapy

Twenty-one trials investigated the effectiveness of Music Therapy; 10 trials are of NHMRC evidence hierarchy II (Clark et al., 1998; Garland et al., 2007; Guétin et al., 2009; Raglio et al., 2008, 2010a, 2010b; Remington, 2002; Sung et al., 2006; Thornley et al., 2016; Tuet and Lam 2006), six trials are of hierarchy III-1 (Chang et al., 2010; Ledger and Baker, 2007; Irish et al., 2006; Suzuki et al., 2004, 2007; van de Winckel et al., 2004), two trials are of hierarchy III-2 (Cooke et al., 2010; Nair et al., 2011), and three trials are of hierarchy IV (Ashida, 2000;
Ray and Mittelman, 2017; Sung and Chang, 2010). The delivery approach, music type, content, session type, duration, frequency of application, and the total intervention time of Music Therapy vary across trials.

The delivery approach can be classified into receptive and active. The $\mathrm{PwD}$ only listened to the music in the receptive approach, while the PwD interacted with the music by singing or playing with instruments in the active approach. The music type can be divided into recorded and live. The contents of the music used were either generic music or music preferred by PwD. The session type was either an individual session or a group session. The duration of music ranged from 10 to 60 minutes in the trials, which reported duration. The reported frequency of application varied from once a week to everyday, and the reported total intervention time spanned from two weeks to one year.

The outcomes of Music Therapy are mainly positive. Seventeen trials demonstrated that one or more BPSD symptoms had reduced significantly (Ashida, 2000; Chang et al., 2010; Clark et al., 1998; Cooke et al., 2010; Garland et al., 2007; Guétin et al., 2009; Raglio et al., 2008, 2010a, 2010b; Ray and Mittelman, 2017; Remington, 2002; Sung et al., 2006; Sung and Chang, 2010; Suzuki et al., 2004, 2007; Tuet and Lam 2006; van de Winckel et al., 2004). Two trials showed that the change in BPSD was insignificant (Irish et al., 2006; Ledger and Baker, 2007), and two trials found that the BPSD symptoms deteriorated after the intervention (Nair et al., 2011; Thornley et al., 2016). Only seven trials considered the auditory capability of the PwD (Chang et al., 2010; Garland et al., 2007; Ray and Mittelman, 2017; Remington, 2002; Sung et al., 2006; Sung and Chang, 2010; van de Winckel et al., 2004). One trial mentioned the music was played "sufficient to be heard throughout the common area," but it did not specify if the music was sufficient to be heard for the researchers or PwD (Nair et al., 2011). Thus, the author decided this trial did not consider the hearing capabilities of PwD.

Aromatherapy

Nine trials have investigated the effectiveness of Aromatherapy; five trials were of NHMRC evidence hierarchy II (Akhondzadeh et al., 2003; Ballard et al., 2002; Fu and Moyle, 2013; Holmes et al., 2002; Smallwood et al., 2001), one trial was of hierarchy III-1 (Snow et al., 2004), and three trials were of hierarchy III-2 (Lin et al., 2007; O'Connor et al., 2013; Yoshiyama et al., 2015). The delivery approach, formulation, concentration, frequency of application, duration, and total intervention time of Aromatherapy varied across trials.

The essential oil was delivered to the PwD through massage, spray, or diffuser. The formulation of 


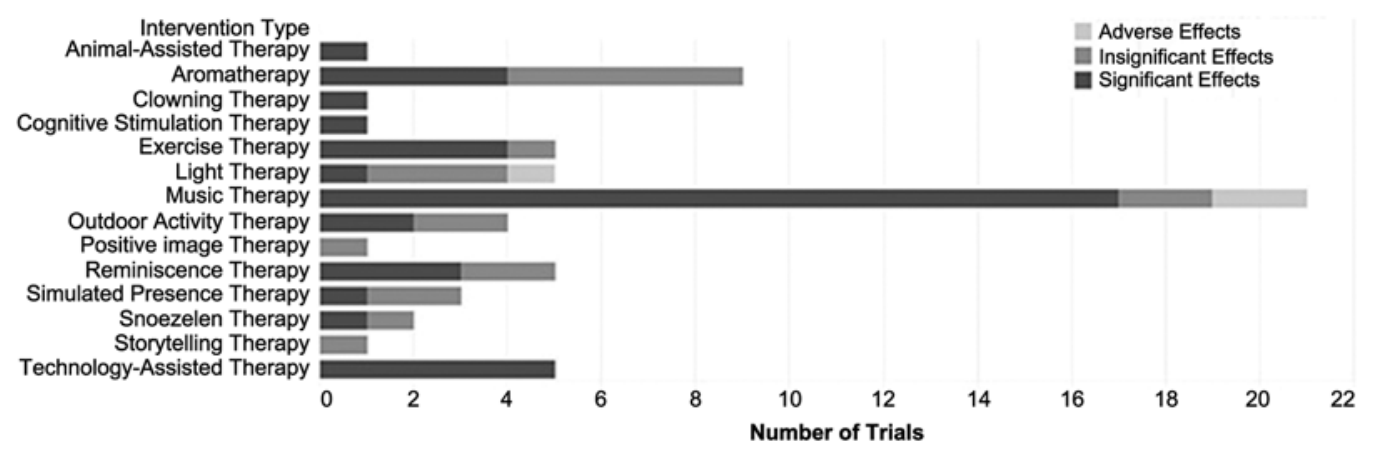

Figure 2. Summary of clinical outcomes of interventions for BPSD in nursing home residents.

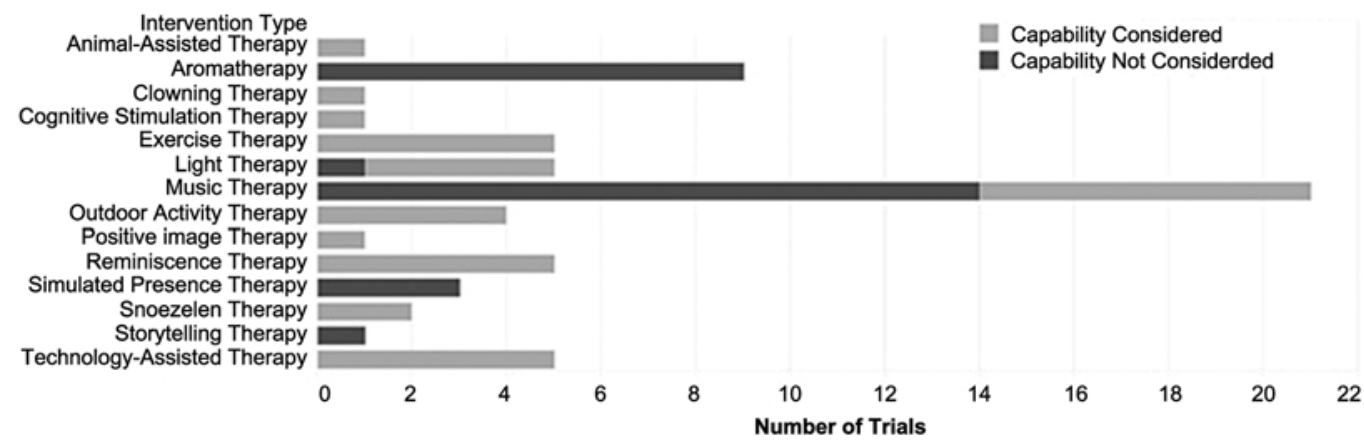

Figure 3. Summary of "capability consideration" of clinical trials on interventions for BPSD in nursing home residents.

essential oil used was either Lavender or Melissa officinalis with concentrations varying from $2 \%$ to $100 \%$. The reported frequency of application ranged from twice daily to three times a week, and the reported duration ranged from two hours to the whole night. The reported total intervention time spanned from ten days to four months.

The outcomes of Aromatherapy are mixed. Four trials showed the BPSD symptoms were significantly alleviated after the intervention, in which the essential oil was applied dermally or via a high-concentration spray (Akhondzadeh et al., 2003; Ballard et al., 2002; Lin et al., 2007; O'Connor et al., 2013). The remaining five trials, in which the essential oil was delivered by spray in low concentration, found no significant reduction in BPSD (Fu and Moyle, 2013; Holmes et al., 2002; Smallwood et al., 2001; Snow et al., 2004; Yoshiyama et al., 2015). None of the trials considered the olfactory capability of PwD.

\section{Light Therapy}

Five trials evaluated the effectiveness of Light Therapy; three trials were of NHMRC evidence hierarchy II (Ancoli-Israel et al., 2003; Burns et al., 2009; Riemersma-van der Lek et al., 2008) and the other two trials were of hierarchy III-1 (Barrick et al., 2010; Hickman et al., 2007). The brightness, duration, and total intervention time of Light Therapy varied across trials.
The reported brightness of the light ranged from 1000 lux to 10,000 lux in the intervention groups and 100 lux to 300 lux in the control groups. The duration of the Light Therapy varied from 120 minutes to a whole day, and the total intervention time spanned from 10 days to 3.5 years.

The outcomes of Light Therapy are mixed. The clinical trial with the best experimental design found that agitation and depression were significantly reduced in a follow-up ranging from 0.5 to 3.5 years. In this trial, bright light was applied for the whole day with or without melatonin in the intervention group, and dim light with placebo in the control group. This is a $2 \times 2$ factorial double-blind cluster-randomized trial comparing two light settings in several nursing homes, with a total of 189 participants, of whom $87 \%$ had dementia (Riemersma-van der Lek et al., 2008). However, three trials found that Light Therapy had no significant effect in reducing BPSD (Ancoli-Israel et al., 2003; Barrick et al., 2010; Burns et al., 2009; Hickman et al., 2007), and one trial reported that the agitation in PwD was worsened (Barrick et al., 2010). One of the five trials did not consider the visual capability of PwD (Ancoli-Israel et al., 2003).

\section{SNOEZELEN Therapy}

Snoezelen Therapy, also referred to as Multisensory Therapy, was investigated by two trials both with NHMRC evidence hierarchy lV (Berkheimer et al., 
2017; van Weert et al., 2005). One trial compared Snoezelen Therapy with Exercise Therapy for three weeks and found that the reduction in agitation was not significant under both intervention types (Berkheimer et al., 2017). The other trial integrated Snoezelen Therapy into 24-hour care for 18 months, with each resident having a personal care plan, and found significant reduction in apathy, aggression, and depression after the intervention (van Weert et al., 2005). Snoezelen Therapy is designed with the sensory capabilities of PwD in mind. Declines in sensory capabilities (i.e. hearing, vision, taste, smell, touch) are common in elderly adults, with the rate of decline varying across senses for each person. By stimulating a few senses together in Snoezelen Therapy, a PwD with sensory impairments in less than five senses could still receive stimuli from the therapy via their remaining functional senses. According to the guidelines for designing perceptible information for PwD, different modes (pictorial, verbal, tactile) should be used together to present essential information (Mäki and Topo, 2009). Hence, all trials applying Snoezelen Therapy have considered sensory capabilities of PwD.

Technology-Assisted Therapy

A Technology-Assisted Therapy is the intervention type in which the roles of caregivers or therapists are replaced by technologies. Five trials were found for this intervention type. One trial explored the effectiveness of a personalized multimedia device (Davison et al., 2016). The device can play music and display images, films, and messages that were selected or made by family members of the PwD. This trial was of NHMRC hierarchy level II and showed a significant reduction in agitation in PwD after the intervention. It also reported that assistance was needed and offered for PwD in late stages. This personalized multimedia device is designed with the touch capabilities of $\mathrm{PwD}$ in mind. Specifically, researchers found some PwD find touch sensitive icons confusing in preliminary field testing, thus they added traditional buttons affixed to the screen for those PwD with that preference.

Four trials examined the effectiveness of a therapeutic robot (Joranson et al., 2015a, 2015b, 2016; Moyle et al., 2017). This robot, with an appearance of a baby seal, was placed on the lap of PwD. The robot generated movement and sound mimicking the baby seal to give somatosensory and auditory stimulations to PwD. The four trials were of NHMRC evidence hierarchy III-1 and reported that agitation and depression in PwD were significantly reduced with no reduction in antipsychotic medication after the intervention. One trial applied the robot in individual sessions (Moyle et al., 2017), and the remaining three trials applied the robot in group sessions (Joranson et al., 2015a, 2015b, 2016). This robot is designed with the sensory capabilities of PwD in mind as it stimulates several senses in $\mathrm{PwD}$, which is similar to Snoezelen therapy. Therefore, all trials in this intervention type have considered the capabilities of PwD.

OTHER SENSORY-ORIENTED INTERVENTIONS Three sensory-oriented interventions were evaluated by one trial only. Animal-Assisted Therapy was examined by a trial of NHMRC evidence hierarchy III-1 (Olsen et al., 2016). This therapy organized regular dog visits in a group session accompanied by a dog handler, which found depression in PwD was reduced significantly after the intervention. Positive Image Therapy was assessed by a trial of hierarchy II (Chou et al., 2016). This therapy displayed images preferred by $\mathrm{PwD}$ with voice prompt during bathing time, which led to fewer behavioral problems. However, the sample size of this trial was too small to calculate the significance. Clowning Therapy was evaluated by a trial of hierarchy IV (Kontos et al., 2016). The elder clowns visited the nursing home regularly to entertain the PwD for 12 weeks and reported that BPSD was reduced significantly after the intervention. In the Animal-Assisted Therapy and Clowning Therapy, several senses of PwD were stimulated, which is similar to Snoezelen Therapy. In the Positive Image Therapy, visual impairment was used as an exclusion criterion in the clinical trial. Therefore, all three trials have considered the sensory capabilities of $\mathrm{PwD}$.

\section{Cognition-oriented therapy}

Reminiscence Therapy

Five trials examined the effectiveness of Reminiscence Therapy, which were all of NHMRC evidence hierarchy level II (Haight et al., 2006; Haslam et al., 2010; Lai et al., 2004; Politis et al., 2004; Wang, 2007). The session type, structure, duration, and total intervention time of Reminiscence Therapy varied across trials. The therapy has been conducted in group or individual sessions via unstructured, semi-structured, and structured activities. The reported duration of one session ranged from 30 to 60 minutes, and the reported total intervention time went from four to eight weeks.

Three trials showed the apathy, agitation, and depression of PwD were significantly reduced after the intervention (Haight et al., 2006; Lai et al., 2004; Politis et al., 2004), and the remaining two trials showed the reduction was insignificant (Haslam et al., 2010; Wang, 2007). This intervention is designed with the memory and attention capabilities of PwD in mind. In terms of memory, this intervention 
stimulates the long-term memory, which is relatively intact in PwD, compared to other types of memories. In terms of attention, this intervention involves interactions between caregivers and $\mathrm{PwD}$ to keep PwD concentrated during the intervention. These interactions are essential as the capability of inhibiting irrelevant information is declined in PwD. According to the guidelines for designing for PwD, the design should remind $\mathrm{PwD}$ about their previous experiences and give feedback immediately, given the short attention span of the PwD (Mäki and Topo, 2009). Thus, the main cognitive capabilities of $\mathrm{PwD}$ were considered in the trials.

\section{Simulated Presence Therapy}

Three trials investigated Simulated Presence Therapy, with one trial of NHMRC evidence hierarchy III-1 (Camberg et al., 1999) and two trials of hierarchy IV (Cheston et al., 2007; Miller et al., 2001). All trials applied audio recordings to simulate a phone call from a family member of the PwD to engage $\mathrm{PwD}$ in conversations.

One trial reported that the response of PwD varied widely from dramatic reduction in distress to apparent indifference (Cheston et al., 2007). One trial found that some $\mathrm{PwD}$ recognized this phone call is a simulation and refused to listen again (Miller et al., 2001). One trial reported that the "interest" subscale of Positive Affect Rating Scale was significantly increased after the intervention; however, there was no change in BPSD (Camberg et al., 1999).

The main cognitive capabilities of PwD were not fully considered in the intervention. In terms of memory, the phone call could trigger the longterm memory of the PwD by the familiar voices. However, in terms of attention, the interactions between PwD and the audio recordings were limited at the audial level, and this lack of sensory engagement could result in the PwD getting easily distracted during the therapy. Besides, it is difficult for caregivers to check if $\mathrm{PwD}$ are distracted. None of the trials have examined if PwD had the adequate capability to inhibit irrelevant information to ensure they can concentrate throughout the therapy. Therefore, these trials have not fully considered the main cognitive capabilities of PwD.

OTHER COGNITION-ORIENTED INTERVENTIONS Two cognition-oriented interventions were examined by one trial only. Cognitive Stimulation Therapy was evaluated by a trial of NHMRC hierarchy II, which found apathy and depression in PwD were reduced significantly after the intervention (Niu et al., 2010). Storytelling Therapy was assessed by a trial of hierarchy III-1, which found no significant reduction in BPSD (Houser et al., 2014). The Cognitive Stimulation Therapy provides cognitive games to stimulate $\mathrm{PwD}$, and its clinical trial considered the cognitive capabilities of PwD by only including PwD in their early to moderate stage (Niu et al., 2010). The Storytelling Therapy prompts a group of PwD to give comments about a picture and uses these comments to form a story, and its clinical trial had no assessment on cognitive capabilities of PwD participants.

\section{Movement-oriented therapy}

Exercise Therapy

Five trials examined the effectiveness of Exercise Therapy; four trials were of NHMRC evidence hierarchy II (Hokkanen et al., 2008; Rolland et al., 2007; Telenius et al., 2015a, 2015b) and one trial was of hierarchy III-1 (Treusch et al., 2015). The content of the exercise varied across trials from intensive strengthening to dance. Ranking the intensity of the exercise in these trials is difficult due to the lack of detailed exercise description in each trial. The total intervention time ranged from 12 weeks to 12 months in these trials.

Four trials found apathy and agitation in PwD reduced significantly (Hokkanen et al., 2008; Telenius et al., 2015a, 2015b; Treusch et al., 2015), while one trial found no significant reduction in BPSD after the intervention (Rolland et al., 2007). All trials in this intervention type considered the movement capabilities of PwD by excluding PwD who were not capable to move independently with or without an assistive device.

\section{Outdoor Activity Therapy}

Four trials examined the effectiveness of Outdoor Activity Therapy; three trials were of hierarchy IV (Calkins et al., 2007; Luk et al., 2011; Vuolo, 2003) and one trial was of hierarchy II (Connell et al., 2007). The content, duration, frequency of application, and total intervention time varied across trials. The reported content included gardening, horticultural therapy, and walking outdoors, and the duration ranged from 30 to 60 minutes. The reported frequency of application went from daily to twice a week, and the total intervention time spanned from 2 weeks to 12 months.

One trial found a significant reduction in verbal agitation in PwD (Connell et al., 2007), and one trial reported the physically non-aggressive behavior in PwD reduced significantly after the intervention (Vuolo, 2003). Two remaining trials found no significant reduction in BPSD (Calkins et al., 2007; Luk et al., 2011). All trials considered the movement capabilities of PwD by only including PwD who 
were capable to move independently or with an assistive device.

\section{Discussion}

As far as we are aware from the current literature, this is the first study to systematically examine the effectiveness of non-pharmacological interventions for BPSD in nursing homes, with an additional assessment criterion based on ergonomics, specifically, capability consideration. The current review investigated whether the capabilities of PwD were considered in the interventions and their clinical trials. The interventions were categorized according to the capabilities of $\mathrm{PwD}$ required to participate, which are sensory-, cognition-, and movement-oriented. The quality of evidence for the effectiveness of these interventions is found to be low in general. Sensoryoriented interventions have been explored and evaluated more than cognition- and movement-oriented interventions, with Music Therapy being investigated by the highest number of trials. In terms of capability consideration, the sensory capabilities have been considered less than cognitive and movement capabilities, especially for auditory and olfactory capabilities.

To elaborate on the capability considerations in clinical trials, the trials satisfied the first "capability consideration" criterion were on Snoezelen Therapy, Technology-Assisted Therapy, Animal-Assisted Therapy, Clowning Therapy, and Reminiscence Therapy. These interventions have accommodated capability considerations in them, thus the trials on these interventions have considered the capabilities in PwD. For interventions without capability considerations (i.e. Music Therapy, Aromatherapy, Light Therapy, Positive Image Therapy, Simulated Presence Therapy, Cognitive Stimulation Therapy, Storytelling Therapy, Exercise Therapy and Outdoor Activity Therapy), it is essential for trials to only include PwD with adequate capabilities for the intervention outcomes to be reliable. From this review, clinical trials on Positive Image Therapy, Cognitive Stimulation Therapy, and all movement-oriented interventions have assessed the capabilities of $\mathrm{PwD}$ for inclusion. Two-thirds of the clinical trials on Music Therapy, one-fifth of the clinical trials on Light Therapy, and all clinical trials on Aromatherapy, Simulated Presence Therapy, and Storytelling Therapy have not assessed the capabilities of PwD for inclusion. This lack of capability consideration implies that not all participants might have had the capabilities that the interventions required. For instance, not all participants can hear the music in a trial on Music Therapy. Consequently, the effectiveness of these interventions would have been underestimated, and, thus, a lack of capability consideration could reduce the quality of a trial and consequently lower the quality of evidence about the corresponding intervention type.

A few factors could cause the sensory capabilities to be considered less than cognitive and movement capabilities. The sensory capabilities are more difficult to measure, and the PwD cannot actively communicate their sensory experiences due to their cognitive impairments. In contrast, the remaining cognitive capabilities of $\mathrm{PwD}$ are relatively welltracked, as they are assessed regularly to monitor how their dementia progresses. The movement capabilities are crucial for the safety of PwD and, thus, were also assessed carefully in movementoriented interventions.

To include capabilities of $\mathrm{PwD}$ in designing interventions and clinical trials, three approaches should be considered. First, clinical trials should include capability requirements in their in- and exclusion criteria and indicate that the capabilities of PwD participants were adequate to participate in the interventions. This practice should be used as a criterion for evaluating the quality of a trial in systematic reviews. Second, a capability profile should be created for each PwD in terms of sensory, cognition, and movement aspects. The suitable interventions for a PwD can then be identified by matching the capability profile with the capability requirements of the interventions. The capabilities of PwD can be tracked over time with this profile to identify new interventions when the current interventions are no longer suitable due to capability decline. This profile also helps researchers to identify suitable PwD for clinical trials. Third, the interventions themselves should be designed with the capabilities of PwD in mind by incorporating design guidelines for $\mathrm{PwD}$ or involving PwD in the design process. An intervention with adequate capability consideration should be able to include as many capability impairments as possible or adapt to the remaining capabilities of PwD. For example, bed-bound exercises could be designed for PwD who are immobile. The third approach is the most fundamental because some capabilities are difficult and costly to measure regularly. Moreover, by designing the interventions with capability considerations, more $\mathrm{PwD}$ with capability impairments will be able to participate and, thus, benefit from the interventions. With more participants, the clinical trials on non-pharmacological interventions for BPSD could have a larger sample size, which could improve the quality of evidence. Since ergonomics has a human-centered approach and has investigated age-related capability changes, it would facilitate the intervention design process.

A meta-analysis is impossible due to the following limitations of the clinical trials. Firstly, the outcomes 
have been measured by a broad range of validated scales and physiological parameters. Over 60 scales have been used with some scales focusing on one aspect of BPSD (e.g., Cohen-Mansfield Agitation Inventory [Cohen-Mansfield, 1997]) while other scales measuring BPSD comprehensively (e.g., Neuropsychiatric Inventory [Kaufer et al., 2000]). The physiological parameters include heart rate, cortisol level, and skin conductance. Comparing the outcomes of these trials is difficult due to this wide variation in measurement methods. In addition, the design of control groups varies across trials. Some trials applied a placebo activity in the control group (e.g., reading activity as a placebo for Music Therapy [Cooke et al., 2010]), while most trials applied "usual care" in the control group. "Usual care" was not clearly described in these trials and further hinders comparison. Moreover, some trials have not accounted for potential confounding factors. For instance, the social interactions between PwD might also contribute to reducing BPSD for Music Therapy conducted in a group session (Sung et al., 2006; Suzuki et al., 2004; van de Winckel et al., 2004). The current study demonstrates a lack of standardization and heterogeneity in study designs of nonpharmacological interventions in clinical trials of BPSD. Therefore, improving data homogeneity, study designs, and reporting in future clinical trials is urgent, which is in accordance with previous systematic reviews (Abraha et al., 2017; Brasure et al., 2016; Cohen-Mansfield, 2001).

The link between the effectiveness of nonpharmacological interventions and their capability considerations cannot be drawn due to the abovementioned limitations in clinical trials. These limitations make comparisons between intervention outcomes across clinical trials difficult. A hypothesis is that interventions that consider capabilities in PwD are more effective than interventions that do not. This hypothesis needs to be examined in future clinical trials; that is, by comparing the outcome measurements of the same intervention with and without capability considerations. This comparison can only be carried out when these clinical trials have overcome the limitations.

Apart from capability considerations, treatment parameters could also affect the effectiveness of the interventions, such as the dose, timing, and duration of an intervention. Cohen-Mansfield stated that the effectiveness of an intervention may depend on these parameters, instead of the inherent applicability of the intervention type (Cohen-Mansfield, 2001). As treatment parameters were not commonly reported in clinical trials, together with the abovementioned limitations in these trials, it is impossible to conclude on how these parameters affected the effectiveness of an intervention, which is a remaining challenge for future studies.

A limitation of this review is that it is only focused on if the capabilities of the PwD are considered in the interventions and their clinical trials. We did not include caregiver interventions in the current study, as the focus of the current review is on capability considerations of PwD. The capabilities of caregivers are also vital for the interventions to be carried out reliably, thus to ensure high quality of evidence. For example, if a caregiver can accomplish the non-pharmacological intervention given the time constraints; and if the training is adequate for the caregiver to understand how to operate a device used for the intervention. However, it is not common for clinical trials to report these details, thus, the consideration of the capabilities of caregivers cannot be made in the current review. This review provides a starting point for considering human capabilities in non-pharmacological interventions and the subsequent clinical trials for BPSD in nursing homes. In future trials, it might be worthwhile to consider the capabilities of both PwD and caregivers. The fact that not all interventions were truly based on capabilities of PwD, and that 28 out of 64 trials did not consider capabilities, is also a limitation of the current review. It would be worthwhile for future studies to explore how to assess if capabilities of PwD are truly considered.

Despite the fact that the current quality of evidence is low, the evidence indicates that a wide variety of non-pharmacological interventions for BPSD in nursing homes have been developed and carried out with a few reported adverse effects. The consideration of human capabilities for antipsychotic medications is not as vital as that of nonpharmacological interventions, which indicates that non-pharmacological interventions are more challenging to be implemented. Given their fewer adverse effects than that of medication, future studies focusing on non-pharmacological interventions for BPSD should include capability considerations for PwD to gather more high-quality evidence.

\section{Recommendations for future research}

The findings lead to the following five recommendations for future studies:

- Capability requirements for PwD should be included in the in- and exclusion criteria of clinical trials on non-pharmacological interventions

- Capability requirements should be included as a criterion for assessing the quality of clinical trials on non-pharmacological interventions in systematic reviews 
- The sensory, cognition, and movement capabilities of each PwD should be assessed regularly over time and recorded in a profile to identify suitable interventions for each PwD and ease the selection process in clinical trials.

- Non-pharmacological interventions should be designed with the capabilities of PwD in mind under the guidance of ergonomics.

- Clinical trials should be conducted more systematically by establishing consensus on outcome measurements, refining study designs, developing reporting standards, and managing confounding factors.

\section{Conflict of interest}

None.

\section{Description of authors' roles}

G. Wang and T. J. M. van der Cammen conceived this study and carried out the systematic review. G. Wang wrote the initial draft of the manuscript. All authors were involved in the critical revisions of the manuscript.

\section{Acknowledgments}

This research was supported by the Chinese Scholarship Council (CSC), China.

\section{Supplementary material}

To view supplementary material for this article, please visit https://www.cambridge.org/core/journals/ international-psychogeriatrics.

\section{References}

Abraha, I., et al. (2017). Systematic review of systematic reviews of non-pharmacological interventions to treat behavioural disturbances in older patients with dementia: the SENATOR-OnTop series. BMF Open, 7, e012759. doi: 10.1136/bmjopen-2016-012759.

Akhondzadeh, S., Noroozian, M., Mohammadi, M., Ohadinia, S., Jamshidi, A. H. and Khani, M. (2003). Melissa officinalis extract in the treatment of patients with mild to moderate Alzheimer's disease: a double blind, randomised, placebo controlled trial. Fournal of Neurology, Neurosurgery, and Psychiatry, 74, 863-866. doi: 10.1136/ jnnp.74.7.863.

Ancoli-Israel, S., etal. (2003). Increased light exposure consolidates sleep and strengthens circadian rhythms in severe Alzheimer's disease patients. Behavioral Sleep Medicine, 1, 22-36. doi: 10.1207/S15402010BSM0101_4.

Ashida, S. (2000). The effect of reminiscence music therapy sessions on changes in depressive symptoms in elderly persons with dementia. Fournal of Music Therapy, 37, 170-182. doi: 10.1093/jmt/37.3.170.

Ballard, C. G., O'Brien, J. T., Reichelt, K. and Perry, E. K. (2002). Aromatherapy as a safe and effective treatment for the management of agitation in severe dementia: the results of a double blind, placebo controlled trial. The fournal of Clinical Psychiatry, 63, 553-558. doi: 10.4088/jcp.v63n0703.

Barrick, A. L., et al. (2010). Impact of ambient bright light on agitation in dementia. International fournal of Geriatric Psychiatry, 25, 1013-1021. doi: 10.1002/gps.2453.

Berkheimer, S. D., Qian, C. and Malmstrom, T. K. (2017). Snoezelen therapy as an intervention to reduce agitation in nursing home patients with dementia: a pilot study. Fournal of the American Medical Directors Association, 18, 1089-1091. doi: 10.1016/j.jamda.2017.09.009.

Brasure, M., et al. (2016). Nonpharmacologic Interventions for Agitation and Aggression in Dementia. Comparative Effectiveness Review No.177. Rockville: Agency for Healthcare Research and Quality. Retrieved from http:// www.ncbi.nlm.nih.gov/pubmed/27099894.

Brodaty, H. and Arasaratnam, C. (2012). Meta-analysis of nonpharmacological interventions for neuropsychiatric symptoms of dementia. American fournal of Psychiatry, 169, 946-953. doi: 10.1176/appi.ajp.2012.11101529.

Burns, A., Allen, H., Tomenson, B., Duignan, D. and Byrne, J. (2009). Bright light therapy for agitation in dementia: a randomized controlled trial. International Psychogeriatrics, 21, 711. doi: 10.1017/ S1041610209008886.

Calkins, M., Szmerekovsky, J. G. and Biddle, S. (2007). Effect of increased time spent outdoors on individuals with dementia residing in nursing homes. Fournal of Housing for the Elderly, 21, 211-228. doi: 10.1300/J081v21n03_11.

Camberg, L., et al. (1999). Evaluation of simulated presence: a personalized approach to enhance well-being in persons with Alzheimer's disease. Fournal of the American Geriatrics Society, 47, 446-452. doi: 10.1111/j.1532-5415 .1999.tb07237.x.

Chang, F.-Y., Huang, H.-C., Lin, K.-C. and Lin, L.-C. (2010). The effect of a music programme during lunchtime on the problem behaviour of the older residents with dementia at an institution in Taiwan. Fournal of Clinical Nursing, 19, 939-948. doi: 10.1111/j.1365-2702.2009 $.02801 . x$.

Cheston, R., Thorne, K., Whitby, P. and Peak, J. (2007). Simulated presence therapy, attachment and separation amongst people with dementia. Dementia, 6, 442-449. doi: 10.1177/14713012070060030703.

Chou, W.-Y., Waszynski, C., Kessler, J., Chiang, Y.-C. and Clarkson, P. J. (2016). Using positive images to manage resistance-to-care and combative behaviors in nursing home residents with dementia: a pilot study. Geriatric Nursing, 37, 215-220. doi: 10.1016/j.gerinurse .2016.02.013.

Clark, M. E., Lipe, A. W. and Bilbrey, M. (1998). Use of music to decrease aggressive behaviors in people with 
dementia. Fournal of Gerontological Nursing, 24, 10-17. doi: 10.3928/0098-9134-19980701-05.

Cohen-Mansfield, J. (1997). Conceptualization of agitation: results based on the Cohen-Mansfield agitation inventory and the agitation behavior mapping instrument. International Psychogeriatrics, 8, 309-315. doi: 10.1017/ S1041610297003530.

Cohen-Mansfield, J. (2001). Nonpharmacologic interventions for inappropriate behaviors in dementia: a review, summary, and critique. The American fournal of Geriatric Psychiatry, 9, 361-381. doi: 10.1097/ 00019442-200111000-00005.

Connell, B. R., Sanford, J. A. and Lewis, D. (2007). Therapeutic effects of an outdoor activity program on nursing home residents with dementia. Fournal of Housing for the Elderly, 21, 194-209. doi: 10.1300/J081v21n03_10.

Cooke, M., Moyle, W., Shum, D. and Harrison, S. (2010). A randomized controlled trial exploring the effect of music on quality of life and depression in older people with dementia. Fournal of Health Psychology, 15, 765-776. doi: $10.1177 / 1359105310368188$.

Czaja, S. J. and Nair, S. N. (2006). Human factors engineering and systems design. In: Handbook of Human Factors and Ergonomics (pp. 32-49). Hoboken, NJ: John Wiley \& Sons, Inc.

Davison, T. E., et al. (2016). A personalized multimedia device to treat agitated behavior and improve mood in people with dementia: a pilot study. Geriatric Nursing, 37, 25-29. doi: 10.1016/j.gerinurse.2015.08.013.

Freudenthal, A. (1999). The Design of Home Appliances for Young and Old Consumers. Ph.D. thesis, Delft: TU Delft-IDE. Retrieved from https://repository.tudelft.nl/islandora/ object/uuid:6c0dd238-b006-436a-893e-252e98ec1475/? collection=research.

Fu, C.-Y. and Moyle, W. (2013). A randomised controlled trial of the use of aromatherapy and hand massage to reduce disruptive behaviour in people with dementia. BMC Complementary and Alternative Medicine, 13, 165. doi: 10.1186/1472-6882-13-165.

Garland, K., Beer, E., Eppingstall, B. and O'Connor, D. W. (2007). A comparison of two treatments of agitated behavior in nursing home residents with dementia: simulated family presence and preferred music. The American fournal of Geriatric Psychiatry, 15, 514-521. doi: 10.1097/01.JGP.0000249388.37080.b4.

Gaugler, J. E., Kane, R. L., Kane, R. A., Clay, T. and Newcomer, R. (2003). Caregiving and institutionalization of cognitively impaired older people: utilizing dynamic predictors of change. The Gerontologist, 43, 219-229. doi: 10.1093/geront/43.2.219.

Gill, S. S., et al. (2007). Antipsychotic drug use and mortality in older adults with dementia. Annals of Internal Medicine, 146, 775. doi: 10.7326/0003-4819-146-11200706050-00006.

Guétin, S, et al. (2009). Effect of music therapy on anxiety and depression in patients with Alzheimer's type dementia: randomised, controlled study. Dementia and Geriatric Cognitive Disorders, 28, 36-46. doi: 10.1159/000229024.

Haight, B. K., Gibson, F. and Michel, Y. (2006). The Northern Ireland life review/life storybook project for people with dementia. Alzheimer's and Dementia, 2, 56-58. doi: $10.1016 /$ j.jalz.2005.12.003.
Haslam, C., Haslam, S. A., Jetten, J., Bevins, A., Ravenscroft, S. and Tonks, J. (2010). The social treatment: the benefits of group interventions in residential care settings. Psychology and Aging, 25, 157-167. doi: 10.1037/a0018256.

Hickman, S. E., et al. (2007). The effect of ambient bright light therapy on depressive symptoms in persons with dementia. Fournal of the American Geriatrics Society, 55, 1817-1824. doi: 10.1111/j.1532-5415.2007.01428.x.

Hokkanen, L., Rantala, L., Remes, A. M., Härkönen, B., Viramo, P. and Winblad, I. (2008). Dance and movement therapeutic methods in management of dementia: a randomized, controlled study. Fournal of the American Geriatrics Society, 56, 771-772. doi: 10.1111/j.1532-5415 .2008.01611.x.

Holmes, C., Hopkins, V., Hensford, C., MacLaughlin, V., Wilkinson, D. and Rosenvinge, H. (2002). Lavender oil as a treatment for agitated behaviour in severe dementia: a placebo controlled study. International fournal of Geriatric Psychiatry, 17, 305-308. doi: 10.1002/gps.593.

Houser, W. S., George, D. R. and Chinchilli, V. M. (2014). Impact of TimeSlips creative expression program on behavioral symptoms and psychotropic medication use in persons with dementia in long-term care: a cluster-randomized pilot study. American fournal of Geriatric Psychiatry, 22, 337-340. doi: 10.1016/j.jagp .2012.12.005.

Irish, M., et al. (2006). Investigating the enhancing effect of music on autobiographical memory in mild Alzheimer's disease. Dementia and Geriatric Cognitive Disorders, 22, 108-120. doi: 10.1159/000093487.

Joranson, N., Pedersen, I. and Mork, A. M. (2015a). Effects of participation in robot-assisted activity groups for nursing home patients with dementia on symptoms of agitation and depression-a cluster-randomized controlled trial. International Psychogeriatrics, 27, S48-S49.

Joranson, N., Pedersen, I., Rokstad, A. M. M. and Ihlebaek, C. (2015b). Effects on symptoms of agitation and depression in persons with dementia participating in robot-assisted activity: a cluster-randomized controlled trial. Fournal of the American Medical Directors Association, 16, 867-873. doi: 10.1016/j.jamda.2015.05.002.

Joranson, N., Pedersen, I., Rokstad, A. M. M. and Ihlebaek, C. (2016). Change in quality of life in older people with dementia participating in Paro-activity: a cluster-randomized controlled trial. Fournal of Advanced Nursing, 72, 3020-3033. doi: 10.1111/jan.13076.

Kales, H. C., Gitlin, L. N. and Lyketsos, C. G. (2015). Assessment and management of behavioral and psychological symptoms of dementia. BMF, 350, h369. doi: 10.1136/BMJ.H369.

Karwowski, W. (2012). The discipline of human factors and ergonomics. In: G. Salvendy (ed.), Handbook of Human Factors and Ergonomics (pp. 1-37) Hoboken: Wiley.

Kaufer, D. I., et al. (2000). Validation of the NPI-Q, a brief clinical form of the neuropsychiatric inventory. The fournal of Neuropsychiatry and Clinical Neurosciences, 12, 233-239. doi: 10.1176/jnp.12.2.233.

Kontos, P., et al. (2016). Elder-clowning in long-term dementia care: results of a pilot study. Fournal of the American Geriatrics Society, 64, 347-353. doi: 10.1111/jgs.13941. 
Lai, C. K. Y., Chi, I. and Kayser-Jones, J. (2004). A randomized controlled trial of a specific reminiscence approach to promote the well-being of nursing home residents with dementia. International Psychogeriatrics, 16, 33-49. doi: 10.1017/S1041610204000055.

Ledger, A. J. and Baker, F. A. (2007). An investigation of long-term effects of group music therapy on agitation levels of people with Alzheimer's disease. Aging $\mathcal{E}$ Mental Health, 11, 330-338. doi: 10.1080/13607860600963406.

Lin, P. W., Chan, W., Ng, B. F. and Lam, L. C. (2007). Efficacy of aromatherapy (Lavandula angustifolia) as an intervention for agitated behaviours in Chinese older persons with dementia: a cross-over randomized trial. International fournal of Geriatric Psychiatry, 22, 405-410. doi: $10.1002 /$ gps. 1688 .

Luk, K. Y., et al. (2011). The effect of horticultural activities on agitation in nursing home residents with dementia. International fournal of Geriatric Psychiatry, 26, 435-436. doi: $10.1002 /$ gps. 2493 .

Mäki, O. and Topo, P. (2009). User needs and user requirements of people with dementia: multimedia application for entertainment. In: Dementia, Design and Technology (pp. 61-75). Amsterdam: IOS Press.

Miller, S., Vermeersch, P. E., Bohan, K., Renbarger, K., Kruep, A. and Sacre, S. (2001). Audio presence intervention for decreasing agitation in people with dementia. Geriatric Nursing, 22, 66-70. doi: 10.1067/mgn .2001 .115200 .

Moyle, W., et al. (2017). Use of a robotic seal as a therapeutic tool to improve dementia symptoms: a cluster-randomized controlled trial. Fournal of the American Medical Directors Association, 18, 766-773. doi: 10.1016/j.jamda .2017.03.018.

Nair, B. K., Heim, C., Krishnan, C., D'Este, C. and Marley, J. (2011). The effect of Baroque music on behavioural disturbances in patients with dementia. Australasian fournal on Ageing, 30, 11-15. doi: 10.1111/j. 1741-6612.2010.00439.x.

NHMRC. (2000). How to use the evidence: assessment and application of scientific evidence Handbook series on preparing clinical practice guidelines. Retrieved from www .ausinfo.gov.au/general/gen_hottobuy.htm.

Niu, Y.-X., et al. (2010). Cognitive stimulation therapy in the treatment of neuropsychiatric symptoms in Alzheimer's disease: a randomized controlled trial. Clinical Rehabilitation, 24, 1102-1111. doi: 10.1177/026921551 0376004.

O'Connor, D. W., et al. (2013). A randomized, controlled cross-over trial of dermally-applied lavender (Lavandula angustifolia) oil as a treatment of agitated behaviour in dementia. BMC Complementary and Alternative Medicine, 13, 315. doi: 10.1186/1472-6882-13-315.

Olsen, C., Pedersen, I., Bergland, A., Enders-Slegers, M.-J., Patil, G. and Ihlebaek, C. (2016). Effect of animal-assisted interventions on depression, agitation and quality of life in nursing home residents suffering from cognitive impairment or dementia: a cluster randomized controlled trial. International fournal of Geriatric Psychiatry, 31, 1312-1321. doi: 10.1002/gps.4436.

Politis, A. M., Vozzella, S., Mayer, L. S., Onyike, C. U., Baker, A. S. and Lyketsos, C. G. (2004). A randomized, controlled, clinical trial of activity therapy for apathy in patients with dementia residing in long-term care. International fournal of Geriatric Psychiatry, 19, 1087-1094. doi: 10.1002/gps.1215.

Raglio, A., et al. (2008). Efficacy of music therapy in the treatment of behavioral and psychiatric symptoms of dementia. Alzheimer Disease E Associated Disorders, 22, 158-162. doi: 10.1097/WAD.0b013e3181630b6f.

Raglio, A., et al. (2010a). Efficacy of music therapy treatment based on cycles of sessions: a randomised controlled trial. Aging $\mathcal{E}$ Mental Health, 14, 900-904. doi: 10.1080/13607861003713158.

Raglio, A., et al. (2010b). Effects of music therapy on psychological symptoms and heart rate variability in patients with dementia. A pilot study. Current Aging Science, 3, 242-246. doi: 10.2174/187460981100 3030242.

Ray, K. D. and Mittelman, M. S. (2017). Music therapy: a nonpharmacological approach to the care of agitation and depressive symptoms for nursing home residents with dementia. Dementia, 16, 689-710. doi: 10.1177/ 1471301215613779.

Remington, R. (2002). Calming music and hand massage with agitated elderly. Nursing Research, 51, 317-323. doi: 10.1097/00006199-200209000-00008.

Riemersma-van der Lek, R. F., Swaab, D. F., Twisk, J., Hol, E. M., Hoogendijk, W. J. G. and Van Someren, E. J. W. (2008). Effect of bright light and melatonin on cognitive and noncognitive function in elderly residents of group care facilities. $\mathcal{F A M A}, 299,2642$. doi: 10.1001/jama .299 .22 .2642 .

Rolland, Y., et al. (2007). Exercise program for nursing home residents with Alzheimer's disease: a 1-year randomized, controlled trial. Fournal of the American Geriatrics Society, 55, 158-165. doi: 10.1111/j.1532-5415 2007.01035.x.

Seppala, L. J., et al. (2018). Fall-risk-increasing drugs: a systematic review and meta-analysis: II. Psychotropics. Fournal of the American Medical Directors Association, 19, 371.e11-371.e17. doi: 10.1016/j.jamda.2017.12.098.

Smallwood, J., Brown, R., Coulter, F., Irvine, E. and Copland, C. (2001). Aromatherapy and behaviour disturbances in dementia: a randomized controlled trial. International fournal of Geriatric Psychiatry, 16, 1010-1013. doi: 10.1002/gps.473.

Snow, L. A., Hovanec, L. and Brandt, J. (2004). A controlled trial of aromatherapy for agitation in nursing home patients with dementia. Fournal of Alternative and Complementary Medicine, 10, 431-437. doi: 10.1089/ 1075553041323696.

Steinberg, M., et al. (2008). Point and 5-year period prevalence of neuropsychiatric symptoms in dementia: the Cache County study. International fournal of Geriatric Psychiatry, 23, 170-177. doi: 10.1002/gps.1858.

Sung, H.-C., Chang, S.-M., Lee, W.-L. and Lee, M.-S. (2006). The effects of group music with movement intervention on agitated behaviours of institutionalized elders with dementia in Taiwan. Complementary Therapies in Medicine, 14, 113-119. doi: 10.1016/j.ctim.2006.03.002.

Sung, H. C. and Chang, A. M. (2010). A preferred music listening intervention to reduce anxiety in older adults with dementia in nursing homes. fournal of Clinical Nursing, 19, 1056-1064. doi: 10.1111/j.1365-2702.2009.03016.x. 
Suzuki, M., Kanamori, M., Nagasawa, S., Tokiko, I. and Takayuki, S. (2007). Music therapy-induced changes in behavioral evaluations, and saliva chromogranin $\mathrm{A}$ and immunoglobulin A concentrations in elderly patients with senile dementia. Geriatrics \& Gerontology International, 7, 61-71. doi: 10.1111/j.1447-0594.2007.00374.x.

Suzuki, M., et al. (2004). Behavioral and endocrinological evaluation of music therapy for elderly patients with dementia. Nursing E Health Sciences, 6, 11-18. doi: 10.1111/j.1442-2018.2003.00168.x.

Telenius, E. W., Engedal, K. and Bergland, A. (2015a). Long-term effects of a 12 weeks high-intensity functional exercise program on physical function and mental health in nursing home residents with dementia: a single blinded randomized controlled trial. BMC Geriatrics, 15, 158. doi: 10.1186/s12877-015-0151-8.

Telenius, E. W., Engedal, K. and Bergland, A. (2015b). Effect of a high-intensity exercise program on physical function and mental health in nursing home residents with dementia: an assessor blinded randomized controlled trial. PloS ONE, 10, e0126102. doi: 10.1371/journal.pone .0126102 .

Thornley, J., Hirjee, H. and Vasudev, A. (2016). Music therapy in patients with dementia and behavioral disturbance on an inpatient psychiatry unit: results from a pilot randomized controlled study. International Psychogeriatrics, 28, 869-871. doi: 10.1017/S10416102 15001866.

Treusch, Y., Majic, T., Page, J., Gutzmann, H., Heinz, A. and Rapp, M. A. (2015). Apathy in nursing home residents with dementia: results from a cluster-randomized controlled trial. European psychiatry. The fournal of the Association of European Psychiatrists, 30, 251-257. doi: 10.1016/j.eurpsy.2014.02.004.

Tuet, R. W. K. and Lam, L. C. W. (2006). A preliminary study of the effects of music therapy on agitation in Chinese patients with dementia. Hong Kong Fournal of Psychiatry, 16, 87-91.

Vuolo, P. (2003). Horticulture therapy in dementia care impact on behavioral symptoms, physical and cognitive activities. Retrieved from https://www.health.ny.gov/ diseases/conditions/dementia/edge/nysdgp/docs/ nysdg_horticulture_therapy_final_report.pdf.

Wang, J.-J. (2007). Group reminiscence therapy for cognitive and affective function of demented elderly in Taiwan. International fournal of Geriatric Psychiatry, 22, 1235-1240. doi: $10.1002 /$ gps.1821.

van Weert, J. C. M., van Dulmen, A. M., Spreeuwenberg, P. M. M., Ribbe, M. W. and Bensing, J. M. (2005). Effects of snoezelen, integrated in $24 \mathrm{~h}$ dementia care, on nurse-patient communication during morning care. Patient Education and Counseling, 58, 312-326. doi: 10.1016/j.pec.2004.07.013.

van de Winckel, A., Feys, H., De Weerdt, W. and Dom, R. (2004). Cognitive and behavioural effects of music-based exercises in patients with dementia. Clinical Rehabilitation, 18, 253-260. doi: 10.1191/02692155 04cr750oa.

Yoshiyama, K., Arita, H. and Suzuki, J. (2015). The effect of aroma hand massage therapy for with dementia. fournal of Alternative and Complementary Medicine, 21, 759-765. doi: 10.1089/acm.2015.0158. 